\title{
The Therapeutic and Preventive Efficacy of Curcumin and Its Derivatives in Esophageal Cancer
}

\author{
Komal Komal', Shilpi Chaudhary², Preeti Yadav³, Raja Parmanik², Mayank \\ Singh $^{2 *}$
}

\begin{abstract}
Esophageal cancer is the eighth most common occurring cancer type worldwide and 6th most common among the cancer related deaths of which the most common type is squamous cell carcinoma which comprise about $90 \%$ of esophageal cancer cases. The standard of care for esophageal cancer is neoadjuvant concurrent chemotherapy and radiation (NACRT) followed by surgery however the prognosis remains dismal with 5 year survival a meager $10-15 \%$. The treatment modalities for esophageal cancer is associated with both long term and short term toxicities. Curcumin has been explored as a therapeutic modality as a chemo adjuvant in different cancers due to its low toxicity profile and potent anticancer effect however despite lot of promising preclinical data it has not progressed from bench side to bed side. The primary reason that has obstructed its application in clinic has been its low bioavailability which was seen in different clinical trials but there has been tremendous progress in developing formulations of curcumin which have significantly increased its bioavailability and are being tested in clinical trials. Esophageal cancer is associated with inflammation that's why curcumin being a natural antioxidant offer a potential avenue to reduce toxicity of current therapeutic modalities in a chemo adjuvant setting while simultaneously targeting different pro oncogenic pathways. The present review tries to cover in depth different aspects of curcumin application in treatment of esophageal cancer and progress of this potent anticancer agent in its treatment and prevention.
\end{abstract}

Keywords: Curcumin- Esophageal cancer- chemoprevention

Asian Pac J Cancer Prev, 20 (5), 1329-1337

\section{Introduction}

Eesophageal cancer prevalence and treatment options

Esophageal cancer signifies the $1 \%$ of total cancer cases (Esophageal Cancer - Cancer Stat Facts n.d.) It is the eighth most common occurring cancer type worldwide and 6th most common among the cancer related deaths (Zheng et al., 2018). There are two histological forms of esophageal cancer. The first type is a squamous cell sarcoma, which is commonly associated with the mid part of the esophagus. The second type is adenocarcinoma which mostly affects the GE junction and the cardia. It mainly occurs in the cells which are associated with the function of fabrication of the mucous. Initially, it leads to a condition called dysplasia (abnormal growth of tissue cells) which eventually results in the cancer of esophagus. There is a wide variation in the incidence and histo-pathological type of esophageal carcinoma in different regions of the world, highlighting the geographical variation of etiological factors for this disease. While squamous cell carcinoma comprises up to $90 \%$ of esophageal cancers in the Eastern hemisphere including the "esophageal cancer belt" (being causally related to smoked and fermented food, hot beverages etc), the adenocarcinomas are the predominant subtype in the West (mostly related to obesity and GERD). Smoking and alcohol consumption contribute to both. Although, it is increasingly being recognized by researchers that these are two distinct disease entities, yet the clinical management of these cases follows a similar paradigm.

Early stage mucosal disease (detected by screening endoscopies, mostly in the Far East) are managed by mucosal resections and endoscopic therapies. But multimodality management including surgery, chemotherapy and radiotherapy is the key to the treatment of locally advanced disease, which comprise the majority of these diseases. The worldwide standard of care for esophageal cancers is Neoadjuvant concurrent chemotherapy and radiation (NACTRT) followed by esophagectomy by a trans-abdominal or combined trans-thoracic and abdominal routes. The GEJ tumors are, however, treated akin to stomach cancers in Europe with perioperative chemotherapy, i.e. Neoadjuvant chemotherapy followed by surgery followed again by adjuvant chemotherapy. The common chemotherapies used 
include Cisplatin, 5-Fluorouracil, Epirubicin, Oxaliplatin and Docetaxel. A good chunk of these patients is metastatic at baseline or recur after some months. Systemic therapy for these patients has made several advances in the recent years specially with the addition of targeted agents for adenocarcinomas like Trastuzumab (anti Her2neu Ab) and Ramucirumab (anti-VEGF Ab), yet the prognosis remains dismal with 5 yr survivals a meager $10-15 \%$. The above treatment paradigms are toxic and associated with high short-term morbidities as well as long term side-effects. In addition, these patients are nutritionally challenged and often with a borderline performance status. There is an unmet need for newer therapeutic modalities to improve outcomes, reduce toxicities and complement the existing therapeutic armamentarium. Paradoxically, no targeted therapies exist for squamous cell carcinomas.

To overcome these severe side effects of chemotherapeutic drugs, phytochemicals are being explored as an alternate treatment option since they have a relatively well-established safety profile but their development from bench side to bed side has lagged behind. Out of diversely explored plant derived secondary metabolites and phytochemicals curcumin and its derivatives are being explored for prevention/ treatment in different cancers. However, their development in esophageal cancer has lagged behind even with the presence of mammoth amount of preclinical data in esophageal cancer.

\section{Curcumin and its derivatives for cancer treatment}

Curcumin (also called diferuloylmethane) is a phytochemical which is actively present in the powdered rhizome of Curcuma longa linn (Aggarwal et al., 2007) Other curcuminoids which are present are demethoxycurcumin and bisdemethoxycurcumin (Sandur et al., 2007). Chemically the molecular weight of this phytochemical is $368.38 \mathrm{~g} / \mathrm{mol}$ and its molecular formula is $\mathrm{C}_{21} \mathrm{H}_{20} \mathrm{O}_{6}$. There are two molecular forms of curcumin - keto form (stable at low $\mathrm{pH}$ and neutral conditions) and enol form (stable in alkaline condition) (Cornago et al., 2008). Column chromatography is a frequently used method for the purification of curcumin from curcumin mix i.e. a mixture of curcumin, demethoxycurcumin and bisdemethoxycurcumin separated by adsorbing the mixture on silica gel using chloroform/dichloromethane and ethanol/methanol as eluents and further purity of curcumin is estimated by high performance liquid chromatography (HPLC) (Paramasivam et al., 2009).

Biologically the therapeutic activities of Curcumin are known since ancient times and its raw formulations been used for a wide variety of metabolic diseases such as diabetes, arthritis and other chronic and inflammatory diseases like allergies and skin conditions. Recently therapeutic potential of Curcumin been investigated for prevention and treatment of various cancers (Anand et al., 2008). Therapeutic efficacy of Curcumin is also being explored more systematically in various inflammatory diseases and cancer as cancer is widely associated with excessive generation of ROS (Reactive oxygen species) but its bioavailability still remains one of the common issues.
Bioavailability of curcumin a roadblock in its development for treatment of esophageal cancer

Although Curcumin is classified as "generally recognized as safe" (GRAS) by the US Food and Drug Administration. The major limitation of the use of curcumin in patient is its poor solubility in aqueous solution and consequently poor bioavailability (Anand et al., 2007)

Bioavailability is the amount of drug which has an active effect when administered in the body. Curcumin though has minimal toxicity, it is still approved by Food \& Drug Administration and world Health Organizer for the consumption has not been widely used in cancer treatment owing to its poor bio-availability (Rahimi et al., 2016) Curcumin is highly insoluble in water because of its hydrophobic nature by the virtue of beta-diketone in center, aromatic rings and tautomeric structure (Grynkiewicz and Ślifirski, 2012). It is seen when 10 or $12 \mathrm{mg} / \mathrm{ml}$ of curcumin is orally administered, it was found that only $50 \mathrm{ng} / \mathrm{ml}$ occurs in the blood plasma which doesn't have high therapeutic effect (Rahimi et al., 2016) Hence, to overcome the limitations, different types of formulations have been designed for the improvement of bio availability of curcumin and nanonization is one of the suitable approaches among all. Some of potentially relevant analogues explored in esophageal cancer are

\section{a. Nano-Curcumin}

Curcumin in its natural free form has low bioavailability and hence has limited efficacy in clinical trials. To overcome this limitation different types of curcumin analogues have been generated to increase solubility of curcumin and hence increase its bio availability. Derivation of the analogs of curcumin is done by dispersing the nanoparticles, namely theracurmin with the natural curcumin to increase its solubility in aqueous solution and, hence increasing its bioavailability. Theracurcumin was used to improve its bioavailability and water solubility (Sasaki et al., 2011) and it is composed of $10 \%$ curcumin, $2 \%$ other curcuminoids (demethoxy curcumin and bisdemethoxy curcumin), 46\%glycerin, $4 \%$ gumghatti, and $38 \%$ water. This compound has shown superior solubility in water and it allows easier in vitro testing, and eventual in vivo administration as compared to free curcumin (Bisht et al., 2007).

\section{b. Asymmetric curcuminoid analogues}

Asymmetric natural analogue of curcuminoid have anti-cancer activity by the virtue of 4-hydroxy-3-methoxy benzene unit. This property of the natural asymmetric curcuminoid is utilized and various analogues are discovered/synthesized which act as therapeutic drugs by downregulating the NF-kB signaling pathway that occurs in gastric cancer cells. Many studies have been done on the effect of asymmetric curcuminoid analogues on gastric cell lines BGC-823, SGC-7901 and MFC cells. It has been found out that the anti-carcinogenic effect of the curcuminoid analogues S01, S02, S04, S06, S07, S12-S14 on BGC-823 cell line is 10 folds greater as compared to that of free curcumin. Similarly, the therapeutic activity of analogues S01-S07, S12-S14 and S04, S06, S07, S12 on 
the cell lines of gastric cancer SGC-7901 and MFC cells is comparatively more than the free curcumin.

Out of all these analogous compounds SO6 is selected as the therapeutic drug for the treatment of gastric cancer because of its strongest inhibitory effect. S06 causes the downregulation of the NF-kB signaling and induces apoptosis of the cancerous cells by the expression of cleaved PARP and upregulation od proapoptotic Bax proteins. It also makes the cells susceptible to the effect of the chemo-preventive drug 'Irinotecan'. A semisynthetic analog of 'camptothecin', that is, Irinotecan induces the cell death in S-phase by inhibiting the topo-1 (Qiu et al., 2017)

\section{c. Curcumin analog P1}

Like all other curcumin analogs, P1 a tropinone curcumin analog also bring the TNF- $\alpha$ induced apoptosis in the Cellosaurus cancer cell lines A549 and HeLa cells by inhibiting the NF-kB signaling pathway. The 10 $\mu \mathrm{mol} / \mathrm{L}$ of P1 doesn't inhibit the binding of NF-kB to DNA but brings the inhibition of NF-kB translocation,

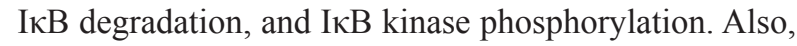
the $1-3 \mu \mathrm{mol} / \mathrm{L}$ amount of $\mathrm{P} 1$ brings the induction of ROS in mitochondria which are reversed by NAC whereas 20 $\mu \mathrm{mol} / \mathrm{L}$ of free curcumin has no effect.(Peng et al., 2013)

\section{d.Curcumin analogue, 3,5-Bis(2-pyridinylmethylidene)-} 4-piperidone (EF31)

EF31 analog of curcumin has a high anticarcinogenic effect than free curcumin. In cancer cell lines its $\mathrm{IC}(50)$ is $5 \mu \mathrm{M}$ of EF31. EF31 inhibit the NF- $\kappa \mathrm{B}$ nuclear translocation and lead to downregulation of the inflammatory cytokine mRNA and proteins such as TN- $\alpha$, IL-1B, IL-6. Also, EF31 IC (50) 1.92 $\mu \mathrm{M}$ inhibit the mechanism of NF- $\kappa B$ signaling by inhibiting the I $\mathrm{B}$ kinase $\beta$ which is more potent than the EF24 $\mathrm{IC}(50) \sim 1.92 \mu \mathrm{M}$ and free curcumin. Hence, EF31 has the more therapeutic efficiency than free curcumin and it's a more promising analog of curcumin for cancer treatment (Olivera et al., 2012).

\section{e. C-5 curcumin analogs}

In chronic myeloid leukemia and colon cancer cell line HCT116 the C5 Curcumin-Fatty Acid conjugates containing either a ten carbon decanoic acid or a sixteen carbon palmitic acid moieties (Sanabria-Ríos et al., 2015) analog of curcumin shows the good cytotoxic effect by inhibiting the TNF- $\alpha$-induced NF- $\kappa \mathrm{B}$ activation in these cancers than the curcumin. Research has been performed on the study of various analogs of C5 curcumin in cancer treatment because of its effective anti-inflammatory properties as compared to curcumin alone (Allegra et al., 2017).

\section{Biological Effects of Curcumin}

Curcumin has a wide range of biological effects due to its anti-inflammatory, antioxidant and anti-carcinogenic properties (Ammon and Wahl, 1991; Afaq et al., 2002). Anti-inflammatory property is by the virtue of methoxy groups in its structure which inhibit the cyclooxygenase-2
(COX-2), Lipoxygenase (LOX) and inducible nitric oxide synthase (iNOS) (Bengmark n.d.). These biological properties of Curcumin advocate it as one of the candidate molecules in the treatment modalities of esophageal cancer. In this review we discuss in detail about the various biological properties and effects of Curcumin formulation in cancer with special focus on esophageal cancer.

\section{a. Anti oxidant property of Curcumin}

Anti-oxidant property of curcumin is based on the presence of o-methoxyphenol and methylenic hydrogen, which donates the electron/hydrogen atom to reactive oxygen species (ROS) and stabilizes it. It also stabilizes the DNA by suppressing the oxidative stress-mediated signaling pathways (Menon and Sudheer, 2007).The antioxidant property of Curcumin is directed towards regulation of ROS metabolisms by the help of anti oxidant and enzymes.

ROS is maintained by the presence of natural antioxidants and enzymes such as superoxide dismutase (SOD), catalase and glutathione peroxidase. In general cancer cells retain a physiological level of these enzymes. However, in many cancers, the level of certain antioxidants enzymes particularly manganese-containing superoxide dismutase (Mn SOD) were found to be dysregulated.

The mitochondrial antioxidant protein MnSOD (Magnesium super oxide dismutase) is a class of enzyme that catalyse dismutation of superoxide into $\mathrm{H}_{2} \mathrm{O}_{2}$ and $\mathrm{O}_{2}$. It has been consider as a tumor suppressor protein (Oberley, 2005) as overexpression of MnSOD inhibits growth in a wide variety of cancer types, including breast cancer (Soini et al., 2001), prostate and brain tumor. In esophageal cancer studies have suggested the role of MnSOD in tumorigenesis. The protein and RNA expression of MnSOD was found to be suppressed in esophageal cancer cells compared to their normal adjacent normal cells (Sun et al., 2011). The effect of Curcumin derived aromatic oil, curcuma has been tested in rat model and it was found that it prevents the transition of esophageal epithelium to Barrett's esophagus and then to esophageal adenocarcinoma by protecting the function of manganese superoxide dismutase (Li et al., 2009). The anti-oxidant and free-radical quenching properties of curcumin plays a key role in inhibiting the carcinogenesis in initial stage. It has inhibitory effects on the production of nitric oxide (NO) and enzymes involved in the oxidation and detoxification of toxic substances as it affects both the Phase I and Phase II enzymes of the hepatic cytochrome p450 enzyme system.

Curcumin has been shown to inhibit the Phase I enzymes that induces the carcinogenic metabolites which may contribute to DNA adduct formation during the oxidation of such substances (Thapliyal and Maru, 2001) On the other hand, curcumin induces the Phase II enzymes involved in detoxification of toxic metabolites which includes glutathione S-transferase, glutathione peroxidase and glutathione reductase (Iqbal et al., 2003). This inhibitory effect of curcumin has been demonstrated in several animal models of oral cancer, mammary carcinoma and intestinal tumors as well (Krishnaswamy 
et al., 1998; Collett et al. 2001; Inano et al., 1999).

\section{b. Effect of Curcumin on cell cycle regulation and apoptosis}

Apoptosis has been considered as an important cellular process and it plays a crucial role in maintaining normal cellular homeostasis (Barry, Behnke, and Eastman 1990) A study suggested that curcumin induces cell death via autophagy, lysosomal activation and increasing ROS (reactive oxygen species) production in HCT 16 colon cancer cells (Wang et al., 2016). Moreover curcumin analogue like (1E, 6E)-1, 7-di (1H-indol-3-yl) hepta-1, 6-diene-3, 5-dione) down-regulated cyclin D1 and activated Caspase 3, 8 and 9 in lung adenocarcinoma (A549), leukemia (K562) colon cancer (SW480) and cervical cancer cells (Sufi et al., 2017; Sha et al., 2016); Singh and Singh, 2009).

Cyclin D1 is one of the regulators of G1 phase which is frequently overexpressed in cancerous tissues and is consider as an oncoprotein because it removes the cell cycle arrest blockade at G1 phase. The expression of Cyclin D1 is frequently amplified in esophageal cancer and it is believed to play a pivotal role in the tumorigenesis and tumor growth. The effect of innovative nano-micelle containing curcumin has been tested on esophageal cancer cell line KYSE-30. Treatment with nano curcumin results in reduced expression of cyclin D1 gene and it was followed by significant increase in cytotoxicity and enhanced anti-proliferation potential in KYSE-30 cell line. Other curcumin derived compounds like nano-curcumin are well-known to induce apoptosis in a variety of cells including pancreatic cancer cells (Sahu-Batra and Srivastava, 2009; Jutooru et al., 2010) and it upregulates the expression of pro-apoptotic members of the Bcl-2 family like Bax and Bak, inhibits the anti-apoptotic Bcl-2 proteins, such as Bcl-XL and Bcl-2 and also found to affect several caspases such as caspase- 8 (Anto et al., 2002; Singh and Singh, 2011). However, the same was not observed in esophageal cell lines. There was no significant change in any above-mentioned pro and anti-apoptotic pathways after treating the EAC cells with nano-curcumin. This might be because EAC cells are known to have intrinsic resistance to apoptosis (O'Sullivan-Coyne et al., 2009). Further, another study suggested that treatment with curcumin did not increase the expression of tumor suppressor micro RNA mir34, which in known to induce apoptosis. In normal condition, p53 induces miR-34a expression thereby leading to cellular apoptosis. However, esophageal cancer cells (and cell lines) are either p53 deficient (TE-7) or have mutant non-functional p53 protein (TE-10) (Barnas et al., 1997). This could be a possible reason why miR-34a is not upregulated in response to curcumin treatment. In cancerous cells, there is an increased level of Nuclear factor- kappa,$(\mathrm{NF}-\mathrm{k} \beta)$ which is related to the high proliferation, angiogenesis, metastasis, anti-apoptotic and chemoresistance in the cancerous cells. There are few reports in the cell line of esophageal adenocarcinoma $\mathrm{OE} 33$ and it was seen that a dose of $50 \mu \mathrm{mol} / \mathrm{L}$ of curcumin caused inhibition of the NF- $\mathrm{k} \beta$ signaling pathway and reduced the level of interleukin (IL8) and prevented the esophageal carcinogenesis (Tian et al., 2008).

\section{c. Anti-proliferative role of Curcumin}

Studies have shown that anti proliferative effect of Curcumin analogs such as nano-curcumin is specific toward cancer but not normal cell lines. However, this effect is not due to the differential cellular uptake of nano-curcumin by cancer cells. This has been proved by fluorescent microscopy as there were equal intra- cellular accumulation in OE19and OE33 (esophageal adenocarcinoma) cell lines as compared to HET-1A (esophageal squamous cells) when treated with same concentration of nano-curcumin. Instead, it

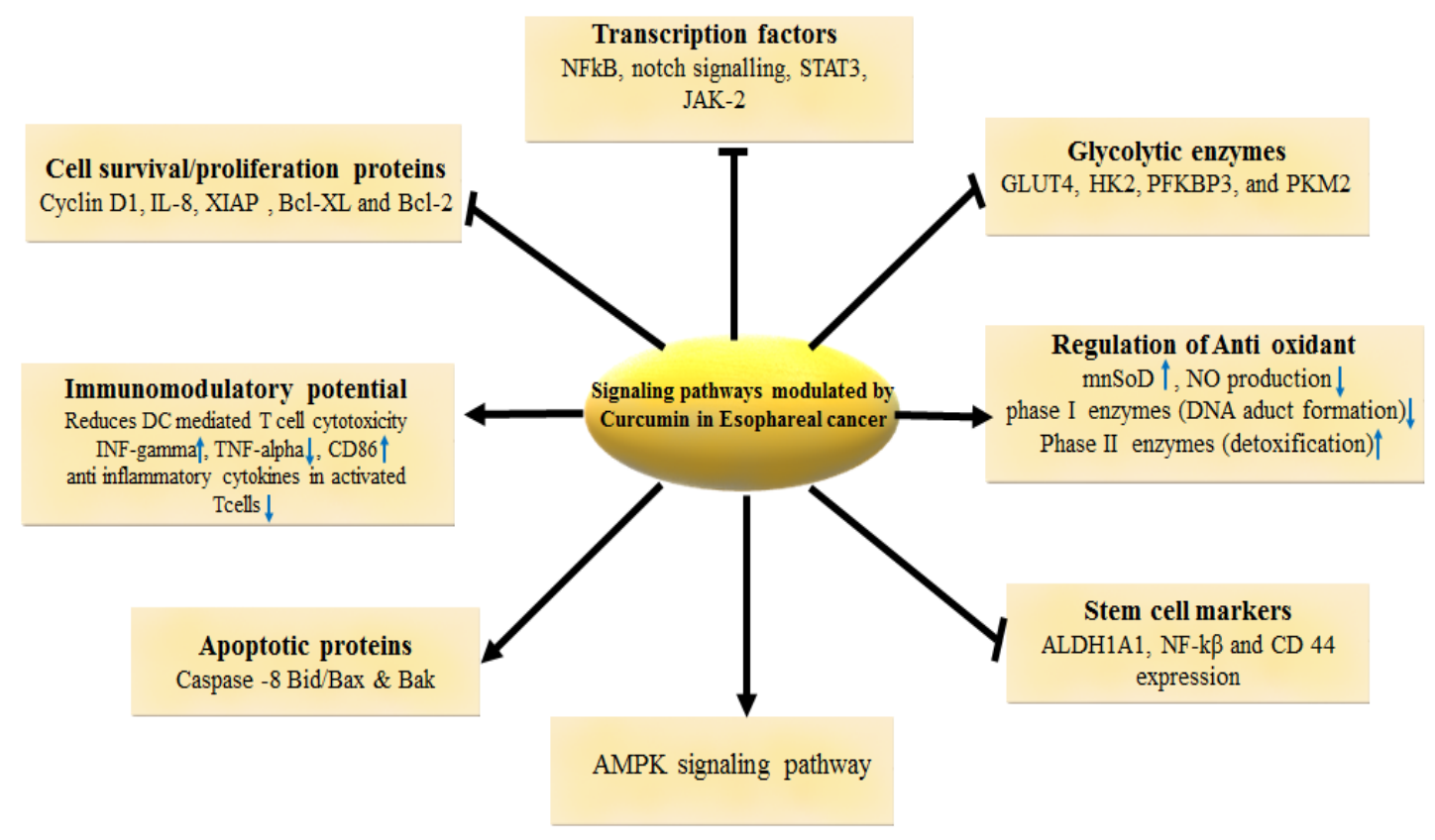

Figure 1. Schematic Figure for the Prominent Molecular Targets of Curcumin in Esophageal Cancer 
could be because of the signaling pathways involved in cellular proliferation are aberrantly activated in cancer cells and make them more susceptible to the effects of nano-curcumin and selectively suppress their proliferation as compared to normal cells which merits its exploration for treatment of esophageal cancer (Milano et al., 2013).

The safety and toxic effects of the use of nano curcumin is being tested in a clinical trial on patients with advance malignancies (ClinicalTrials. gov identi-fier: NCT01201694) The patient group included all the advanced stages of cancers and except one single event of diarrhea, no adverse events have been recorded. Other clinical trials \{NCT 00027495 (Colon cancer), NCT 00969085 (Cutaneous T-cell lymphoma), NCT 00094445 (Advanced pancreatic cancer) $\}$ with various compositions of Curcumin has showed promised results Except one single event of diarrhea, no adverse events have been recorded. This suggests that this form of nano-curcumin is safe and well tolerated (ClinicalTrials.gov identifier: NCT01201694; (Kanai et al., 2012) however; its efficacy in treatment of esophageal cancer remains to be explored.

\section{d. Immunomodulatory potential of Curcumin}

It has been observed that nano-curcumin has a sensitizing effect on DC-mediated T cell cytotoxicity and leads to increasing cell lysis on EAC cells. Probable mechanism behind this would be after activation by the DCs, T cells become effector cytotoxic T lymphocytes (CTLs), which can recognize and lyse the tumor cells. This suggests that nano-curcumin can potentiate the immune response to the EAC cells. Treatment with Thera curcumin increases esophageal tumor cells susceptibility to be killed by cytotoxic T cells (Milano et al., 2013)

It was found that nano-curcumin also increases the IFN- $\gamma$ secretion and decreased the TNF- $\alpha$ secretion in the co-culture of OE33 with CTLs and nano-curcumin (Milano et al. 2013). Along with this nano-curcumin up-regulates the expression of the co-stimulatory molecule CD86 and reduced the levels of anti-inflammatory cytokines in activated T cells (Bisht et al., 2007) Hence, Nano-curcumin along with DC immunotherapy have strong anticancer effect and can be exploited in future for cancer treatments. All these studies propose that this compound is extremely attractive to be used in immune combinatorial therapies for EAC. However, further in vivo evaluations are warranted to confirm its efficacy.

\section{Cell signaling pathways regulated by Curcumin and its analogs}

Curcumin and its biological analogs play a key role in altering several cell signaling pathways directly or indirectly. Some of the major pathways investigated to explore the application of Curcumin as therapeutic candidates include NF-k $\beta$, TGF- $\beta$, Notch, STAT and AMPK.

\section{a. NF-kB pathway}

In majority of cancer cells, there is an increased level of Nuclear factor- kappaB ( NF- $\kappa \mathrm{B}$ ) signaling pathway is seen. This is related to the high proliferation, angiogenesis, metastasis and chemoresistance in the cancerous cells.
Treatment with Curcumin was found to negatively regulate the NF- $\mathrm{KB}$ transcription factor by inhibition of Notch signaling which is involved in cell proliferation, apoptosis, maintenance of stem cell and their renewal. Down regulation of NF- $\kappa \mathrm{B}$ factor results in a considerable decrease in expression of NF- $\kappa \mathrm{B}$ regulated genes, which includes Bcl-2, cyclin D1 as well as vascular endothelial growth factor among many others (O'Riordan et al., 2005). Reactive oxygen species mediated damage and overexpression of antiapoptotic genes are responsible for the pathogenesis of Barrett's esophagus, NF- $\kappa \mathrm{B}$ activation leads to upregulation of anti-apoptotic genes and IL-8 (Abdel-Latif et al., 2004). Increased IL-8 is associated with aggressive, highly proliferative and immuno-suppressed tumor type (Hidaka et al., 2002) and has been shown to be elevated at 4-fold higher level in Barrett's epithelium and esophagus adenocarcinoma (Jenkins et al., 2007; O'Riordan et al., 2005) tissues than in matched squamous tissue.

Curcumin treatment reverse this effect on esophageal OE33 cell line as its exposure blocks the NF- $\kappa B$ pathway and subsequent anti apoptotic genes like BCL-XL, XIAP and IL8 levels. At the tissue level there was up-regulation of $\mathrm{I}-\mathrm{\kappa B}$ expression in Barrett's tissues in the curcumin-supplemented esophageal epithelial cells (HET-1A) (Rafiee et al., 2009). Down regulation of anti-apoptotic genes leads to apoptosis of the cells with damaged DNA which was induced by deoxycholic acid in vitro (Rawat et al., 2012) Furthermore, a pilot study in patients with barrette esophagus showed a trend which suggests that abrogation of IL-8 expression as well as significantly increased levels of apoptosis in the curcumin arm (Bhattacharyya et al., 2010). To sum it all curcumin pretreatment inhibits the NF- $\kappa \mathrm{B}$ mediated pathways and clear out the damaged DNA containing cells by inducing apoptosis and hence prevents transformation of barrette esophagus to esophagus adenocarcinoma.

\section{b. TGF- $\beta$ and Notch pathway}

The interplay between the TGF- $\beta$ and Notch pathway is critical in the transformation of esophageal stem cells. TGF- $\beta$ signaling is implicated in cell-cycle control, differentiation, and modulation of a number of cancers, particularly of the gastrointestinal tract (Tang et al., 2003; Mishra et al., 2005). Various TGF- $\beta$ signaling intermediates were found to be dysfunctional in Barrett's associated adenocarcinoma. Loss of heterozygosity of Smad4 (Ch 18q21.1) was associated with $45 \%$ of cases whereas low mRNA levels of TBRII have been associated with $27 \%$ of Barrett's adenocarcinoma. Dysfunction of TGF- $\beta$ signaling leads to aberrant activation of Notch signaling pathway which plays a critical role in the processes of tumor cell proliferation, apoptosis and stem cells maintenance and is closely associated with tumorigenesis in esophageal cancer cell. It was found that treatment with curcumin down regulates the notch signaling pathway and its c-secretase complex proteins. This leads to inhibition of the proliferative potential of esophageal cancer cells as it suppresses the formation of esophageal cancer cell colonies and inhibits esophagosphere formation. In addition to inhibition of 
Notch-1 and its ligand Jagged-1, curcumin also inhibits of the Notch-1 downstream target Hes-1 in esophageal cancer cells. Hes-1 is an important notch signaling target and mediator (Subramaniam et al., 2012) Its expression is up-regulated in Barrett's associated adenocarcinoma tissues as well as in esophageal adenocarcinoma cells and highly up-regulated in all adenocarcinoma cell lines. Further downregulation of notch signaling with the treatment of curcumin effectively promotes cell cycle arrest, apoptosis and also inhibits oncomir miRNA expression and induces tumor suppressor miRNA expression. Inhibitors of dysfunctional of TGF- $\beta$ signaling or Notch inhibitors like curcumin could hold promise for new personalized therapeutic approaches in esophageal adenocarcinoma (Mendelson et al., 2011).

\section{c. STAT signaling pathway}

Signal transducers and activators of transcription (STATs) mediated interferon-dependent gene expression $(\mathrm{Fu}, 1992)$ contribute to proliferation, uncontrolled differentiation, survival, invasion, tumorigenesis, and resistance to chemotherapy (Buettner et al., 2002; Yu and Jove, 2004; Huynh et al., 2017) and is considered a key regulator of human cancers. Activation of STAT3 plays a major role in cell growth and survival as numerous genes like Bcl-2, Bcl-xL, cyclins D1/D2, BIRC5, CDKN1A, MCL-1, and c-MYC are downstream targets of STAT3 (Yu et al. 2014) (Haura et al 2005). Upregulation of STAT3 has found in majority of cancer including esophageal carcinoma (Tian et al., 2017) and is consider as an ideal target for cancer therapy.

The effect of curcurmin analogue 2-pyridyl cyclohexanone was investigated on the proliferation of ESCC cells and found that treatment of human ESCC cell lines with it promoted loss of both p-STAT3 and p-JAK2. Further treatment with 2-pyridyl cyclohexanone decreased mitochondrial membrane potential in the Eca109 and EC9706 cells and induces apoptosis by suppressing $\mathrm{Bcl}-2$ and $\mathrm{Bcl}-\mathrm{xL}$ expression, whereas promoted $\mathrm{Bid} / \mathrm{Bax}$ expression in a dose dependent manner. In conclusion, curcumin analog 2-pyridyl cyclohexanone decreases basal STAT3 phosphorylation and promotes apoptosis of ESCC cells (Wang et al., 2018).

\section{d. AMPK signaling pathway}

Cancer cells shift their metabolism towards glycolysis to support the biosynthetic demands which is necessary to sustain their proliferation, adapt to stress, and support in the adaptation to microenvironment (Obre and Rossignol 2015; Soga, 2013). Several glycolytic enzymes are considered as oncogene as their level was upregulated to support high metabolic byproduct and energy demands of cancer cells. Treatment with natural curcumin down-regulates the mRNA and protein expression of key glycolytic enzymes, including GLUT4, HK2, PFKBP3, and PKM2 in Ec109 human esophagus cancer cells. It induces a metabolic switch by inhibiting the Warburg effect in Ec109 cells and activates AMPK signaling pathway. AMPK functions as an energy sensor in cancer and regulates cell growth and proliferation in response to stress by regulating cellular metabolic pathways (Faubert et al., 2015; Kim and He, 2013) Collectively curcumin reversed Ec109 cell energy replenishment to overcome metabolic stress and inhibited cell growth and inhibited the Warburg effect by activating the AMPK signaling pathway (Zhang et al., 2015).

\section{Effect of Curcumin on cancer stem cell (CSCs)}

Resistance to chemo-radiation therapy has emerged as a main cause of relapses in many cancers. This phenomenon arises due to presence of subpopulations of cancer cells known as cancer stem cells (CSCs). Therapeutic agents that can target CSCs are essential for the treatment of cancer. The effect of curcumin on CSCs have been evaluated in different cancer cell lines TE-1, TE-8, KY5, KY-10, YES-1, and YES-2 cells derived from human esophageal squamous cell carcinomas. After treatment of these cells with $20-80 \mu \mathrm{M}$ curcumin for $30 \mathrm{hrs}$, cancer stem cell markers like level of ALDH1A1, NF-k $\beta$ and expression of CD 44 were evaluated in curcumin surviving subpopulation. It was found that curcumin-surviving cell lines contain fewer stem-like markers (Almanaa et al., 2013). Curcumin mediates its anti-carcinogenic properties by causing hindrance in the signaling pathways of the cancer stem cells such as Wnt, Notch, Signal transducers and activator (STAT) and interleukin-8 (Norris et al., 2013). Another study by the same group suggested that curcumin at $60 \mu \mathrm{M}$ prevented secondary sphere formation from tumorsphere (Almanaa et al., 2013). It was found that treatment with low concentration i.e. $40 \mu \mathrm{M}$ produced irregularly shaped spheres which suggested that cell interactions or extracellular matrix through which CSCs communicate may be disrupted. Another study supported that curcumin treatment significantly reduces both primary and secondary sphere formation in a dose dependent manner in two ESCCs (TE-7 and TE-10) esophageal cancer cell lines (Almanaa et al., 2012). Collectively there is ample data to indicate that there was a decrease in the CSCs in recurrent esophageal tumor after curcumin treatment.

Curcumin in combination therapy for esophageal cancer

Combination of curcumin with other therapeutic agents have been tested in many solid tumors including lung cancer, prostate, hepatic, gastric cancer etc. Combining curcumin with EGCG (epigallocatechin-3-gallate), lovastatin, significantly reduces the viability and invasion capacity of esophageal cancer cells in vitro. At the molecular level, these three agents in combination inhibited the expression of phosphorylated Erk1/2, c-Jun, COX-2 and induced caspase 3 expression in esophageal cancer cells in vitro and in nude mice xenograft model. It was hypothesized that combination of curcumin with other drugs may targets phosphorylated Erk1/2,c-jun and Cox-2 protein and effectively inhibits esophageal cancer cells (Ye et al., 2012). Curcumin with 5-FU and p65 siRNA inhibit the phosphorylation of $\mathrm{IkB} \alpha$ and expression of $\mathrm{p} 65$ gene and in turn inhibits NF-kB signaling pathway and causes the apoptosis of the cancerous cells and work as an important factor in the treatment in the Cellosaurus cell line EC9706 and Eca109 (Tian et al., 2012). 


\section{Conclusions and future perspectives}

Since ancient time, natural products have often been used to treat variety of diseases and injuries. There is mammoth amount of data available which underline the efficacy of curcumin in treatment of different types of cancer and its underlying preventive value in controlling the process of carcinogenesis however the progress from bench side to bedside has not been easy for this widely available and relatively safe drug due to its poor bioavailability. The need of today is to explore ways to alter its bioavailibity at site of tumor. Different derivatives of curcumin are in development stage in different cancers including esophageal cancer and the future research in this field be focused on testing efficacy of these novel compounds and finally taking them to clinical trials, so they can be used as a mono or adjuvant therapy. These modern Curcumin formulations should be thoroughly examined by well-designed clinical trials, which will open the path for newer drug and therapeutics options for the treatment of esophageal cancer.

\section{References}

Abdel-Latif MM, O'Riordan J, Windle HJ, et al (2004). NF-KappaB activation in esophageal adenocarcinoma: relationship to Barrett's metaplasia, survival, and response to Neoadjuvant Chemoradiotherapy. Ann Surg, 239, 491-500.

Afaq F, Vaqar MA, Nihal A, Hasan M (2002). Botanical antioxidants for chemoprevention of photocarcinogenesis. Front Biosci, a journal and virtual library 7: d784-92. http:// www.ncbi.nlm.nih.gov/pubmed/11897547

Aggarwal BB, Sundaram C, Malani N, Ichikawa H (2007) "CURCUMIN: THE INDIAN SOLID GOLD." In the molecular targets and therapeutic uses of curcumin in health and disease, Boston, MA: Springer US, pp 1-75. http://www. ncbi.nlm.nih.gov/pubmed/17569205

Allegra A, Innao V, Russo S, et al (2017). Anticancer activity of curcumin and its analogues: Preclinical and clinical studies. Cancer Invest, 35, 1-22.

Almanaa TN, Geusz ME, Jamasbi RJ. (2012). Effects of curcumin on stem-like cells in human esophageal squamous carcinoma cell lines. BMC Complement Altern Med, 12, 1180.

Almanaa TN, Geusz ME, Jamasbi RJ (2013). A new method for identifying stem-like cells in esophageal cancer cell lines. Cancer, 4. http://www.jcancer.org

Ammon H, Wahl M (1991). Pharmacology of curcuma longa. Planta Med, 57, 1-7.

Anand P, Sundaram C, Jhurani S, Kunnumakkara AB, Aggarwal BB (2008). Curcumin and cancer: An 'old-age' disease with an 'age-old' solution. Cancer Lett, 267, 133-64.

Anand P, Kunnumakkara AB, Newman RA, Aggarwal BB (2007). Bioavailability of curcumin: Problems and promises. Mol Pharm, 4, 807-18.

Anto RJ, Mukhopadhyay A, Denning K, Aggarwal BB (2002). Curcumin (Diferuloylmethane) induces apoptosis through activation of caspase-8, BID cleavage and cytochrome c release: Its suppression by ectopic expression of $\mathrm{Bcl}-2$ and Bcl-X1. Carcinogenesis, 23, 143-50.

Barnas C, Planche MG, Furukawa Y, et al (1997). Inactivation of the $\mathrm{P} 53$ protein in cell lines derived from human esophageal cancers. Int J Cancer, 71, 79-87.

Barry MA, Behnke CA, Eastman A (1990). Activation of programmed cell death (apoptosis) by cisplatin, other anticancer drugs, toxins and hyperthermia. Biochem
Efficacy of Curcumin as a Chemoadjuvant in Esophageal Cancer

Pharmacol, 40, 2353-62.

Bengmark S (2006). Curcumin, an atoxic antioxidant and natural NFkappaB, cyclooxygenase-2, lipooxygenase, and inducible nitric oxide synthase inhibitor: A shield against acute and chronic diseases. JPEN J Parenter Enteral Nutr, 30, 45-51.

Bhattacharyya S, Hossain MS, Mohanty D, et al (2010) Curcumin reverses $\mathrm{T}$ cell-mediated adaptive immune dysfunctions in tumor-bearing hosts. Cell Mol Immunol, 7, 306-15.

Bisht S, Feldmann G, Soni S, et al (2007) Polymeric nanoparticleencapsulated curcumin: A novel strategy for human cancer therapy. J Nanobiotechnol, 5, 3.

Buettner R, Mora LB, Jove R (2002). Activated STAT signaling in human tumors provides novel molecular targets for therapeutic intervention. Clin Cancer Res, 8, 945-54.

Collett GP, Robson CN, Mathers JC, Campbell FC (2001). Curcumin modifies Apc(Min) apoptosis resistance and inhibits 2-Amino 1-Methyl-6-Phenylimidazo[4,5-b]Pyridine (PhIP) induced tumour formation in Apc(Min) mice. Carcinogenesis, 22, 821-5.

Cornago P, Claramunt RM, Bouissane L, Alkorta I, Elguero J (2008). A study of the Tautomerism of $\beta$-Dicarbonyl compounds with dpecial emphasis on curcuminoids. Tetrahedron, 64, 8089-94.

Faubert B, Vincent EE, Poffenberger MC, Jones RG (2015). The AMP-activated protein kinase (AMPK) and cancer: Many faces of a metabolic regulator. Cancer Lett, 356, 165-70.

$\mathrm{Fu}$ XY (1992). A transcription factor with SH2 and SH3 domains is girectly activated by an interferon alpha-induced cytoplasmic protein tyrosine kinase(S). Cell, 70, 323-35.

Grynkiewicz G, Ślifirski P (2012). Curcumin and curcuminoids in quest for medicinal status. www.actabp.pl.

Haura EB, Turkson J, Jove R. (2005) Mechanisms of disease: insights into the emerging role of signal transducers and activators of transcription in cancer. Nat Clin Pract Oncol, 2, 315-24.

Hidaka H, IshikoT, Furuhashi T, et al (2002). Curcumin inhibits interleukin 8 production and enhances Interleukin 8 receptor expression on the cell surface. Cancer, 95, 1206-14.

Huynh J, Etemadi N, Hollande F, et al (2017). The JAK/STAT3 Axis: A comprehensive drug target for solid malignancies. Sem Cancer Biol, 45, 13-22.

Inano H, Onoda M, Inafuku N, et al (1999). Chemoprevention by curcumin during the promotion stage of tumorigenesis of mammary gland in rats irradiated with Gamma-Rays. Carcinogenesis, 20, 1011-18.

Iqbal M, Sharma SD, Okazaki Y, Fujisawa M, Okada S, (2003). Dietary supplementation of curcumin enhances antioxidant and phase II metabolizing enzymes in DdY male mice: Possible role in protection against chemical carcinogenesis and toxicity. Pharm Toxicol, 92, 33-8.

Jenkins G, Mikhail J, Alhamdani A, et al (2007). Immunohistochemical study of nuclear factor-KappaB activity and interleukin- 8 abundance in oesophageal adenocarcinoma; a useful strategy for monitoring these biomarkers. J Clin Pathol, 60, 1232-37.

Jutooru I, Chadalapaka G, Lei P, and Safe S, (2010). Inhibition of NFkappaB and pancreatic cancer cell and tumor growth by curcumin is dependent on specificity protein down-regulation. J Biol Chem, 285, 25332-44.

Kanai M, Imaizumi A, Otsuka Y, et al (2012). Dose-escalation and pharmacokinetic study of nanoparticle curcumin, a potential anticancer agent with improved bioavailability, in healthy human volunteers. Cancer Chem Pharmacol, 69, 65-70.

Kim IY, He YY (2013). Targeting the AMP-activated protein kinase for cancer prevention and therapy. Front Oncol, 3, 175.

Asian Pacific Journal of Cancer Prevention, Vol 201335 
Krishnaswamy K, Goud VK, Sesikeran B, Mukundan MA, Krishna TP (1998). Retardation of experimental tumorigenesis and reduction in DNA adducts by turmeric and curcumin. Nutr Cancer, 30, 163-6.

Li Y, Wo JM, Liu Q, Li X, Martin RC (2009). Chemoprotective effects of curcuma aromatica on esophageal carcinogenesis. Ann Surg Oncol, 16, 515-23.

Mendelson J, Song S, Li Y, et al (2011). Dysfunctional transforming Growth factor- $\beta$ signaling with constitutively active notch signaling in Barrett's esophageal adenocarcinoma. Cancer, 117, 3691-3702.

Menon VP, Sudheer A (2007). Antioxidant and anti-inflammatory properties of curcumin. In the molecular targets and therapeutic uses of curcumin in health and disease, Boston, MA: Springer US, pp 105-25.

Milano F, Mari L, Luijtgaarden VW, et al (2013). Nano-curcumin inhibits proliferation of esophageal adenocarcinoma cells and enhances the $\mathrm{T}$ cell mediated immune response. Front Oncol, 3, 137.

Mishra L, Derynck R, Mishra B (2005). Transforming growth factor-beta signaling in stem cells and cancer. Science (New York, N.Y.), 310, 68-71.

Norris L, Karmokar A, Howells L, et al (2013). The role of cancer stem cells in the anti-carcinogenicity of curcumin. Mol Nutr Food Res, 57, 1630-37.

O'Riordan JM, Abdel-latif MM, Ravi N (2005). Proinflammatory cytokine and nuclear factor Kappa-B expression along the inflammation-metaplasia-dysplasia-adenocarcinoma sequence in the esophagus. Am J Gastroenterol, 100, 1257-64.

O'Sullivan-CG, O'Sullivan GC, O'Donovan TR, Piwocka K, McKenna SL (2009). Curcumin induces apoptosis-independent death in oesophageal cancer cells. Br J Cancer, 101, 1585-95.

Oberley LW (2005). Mechanism of the tumor suppressive effect of MnSOD overexpression. Biomed Pharmacother, 59, 143-48.

Obre E, Rossignol R (2015). Emerging concepts in bioenergetics and cancer research: metabolic flexibility, coupling, symbiosis, switch, oxidative tumors, metabolic remodeling, signaling and bioenergetic therapy. Int J Biochem Cell Biol, 59, 167-81.

Olivera A, Moore TW, Hu F, et al (2012). Inhibition of the NF-KB signaling pathway by the curcumin analog, 3,5-Bis(2-Pyridinylmethylidene)-4-Piperidone (EF31): Anti-inflammatory and anti-cancer properties. Int Immunopharmacol, 12, 368-77.

Paramasivam M, Poi R, Banerjee H, Bandyopadhyay A, (2009). Food chemistry high-performance thin layer chromatographic method for quantitative determination of curcuminoids in curcuma longa germplasm. Applied Science Publishers. http://agris.fao.org/agris-search/search. do? recordID $=$ US201301553806.

Peng Y, Zheng J, Zhou Y, Li J (2013). Characterization of a novel curcumin analog $\mathrm{P} 1$ as potent inhibitor of the NF-KB signaling pathway with distinct mechanisms. Acta Pharmacol Sinica, 34, 939-50.

Qiu P, Zhang S, Zhou Y, et al (2017). Synthesis and evaluation of asymmetric curcuminoid analogs as potential anticancer agents that downregulate NF-KB activation and enhance the sensitivity of gastric cancer cell lines to irinotecan chemotherapy. Eur J Med Chem, 139, 917-25.

Rafiee P, Victoria NM, Manley S, et al (2009). Effect of curcumin on acidic PH-induced expression of IL-6 and IL-8 in human esophageal epithelial cells (HET-1A): Role of PKC, MAPKs, and NF-KB. Am J Physiol Gastrointest Liver Physiol, 296, 388-98.
Rahimi HR, Nedaeinia R, Sepehri SA, et al (2016). Novel delivery system for natural products: Nano-curcumin formulations. Avicenna J Phytomed, 6, 383-98.

Rawat N, Alhamdani A, McAdam E, et al (2012). Curcumin abrogates bile-induced NF-KB activity and DNA damage in vitro and suppresses NF-KB activity whilst promoting apoptosis in vivo, suggesting chemopreventative potential in Barrett's oesophagus. Clin Transl Oncol, 14, 302-11.

Sahu RP, Batra S, Srivastava SK (2009). Activation of ATM/ Chk1 by curcumin causes cell cycle arrest and apoptosis in human pancreatic cancer cells. Br J Cancer, 100, 1425-33.

Sanabria-Ríos DJ, Rivera-Torres Y, Rosario J, et al (2015). Synthesis of novel C5-curcuminoid-fatty acid conjugates and mechanistic investigation of their anticancer activity. Bioorg Med Chem Lett, 25, 2174-80.

Sandur SK, Pandey MK, Sung BA, et al (2007). Curcumin, demethoxycurcumin, bisdemethoxycurcumin, tetrahydrocurcumin and turmerones differentially regulate anti-inflammatory and anti-proliferative responses through a ROS-independent mechanism. Carcinogenesis, 28, 1765-73.

Sasaki H, Sunagawa Y, Takahashi K, et al (2011). Innovative preparation of curcumin for improved oral bioavailability. Biol Pharma Bull, 34, 660-65.

Sha J, Li J, Wang W, et al (2016). Curcumin induces G0/G1 arrest and apoptosis in hormone independent prostate cancer DU-145 cells by down regulating notch signaling. Biomed Pharm, 84, 177-84.

Singh M, Singh N (2009). Molecular mechanism of curcumin induced cytotoxicity in human cervical carcinoma cells. Mol Cell Biochem, 325, 107-19.

Singh M, Singh N (2011). Curcumin counteracts the proliferative effect of estradiol and induces apoptosis in cervical cancer cells. Mol Cell Biochem, 347, 1-11.

Soga T (2013). Cancer metabolism: Key players in metabolic reprogramming. Cancer Sci, 104, 275-81.

Soini Y, Vakkala M, Kahlos K, Pääkkö P, Kinnula V (2001). MnSOD expression is less frequent in tumour cells of invasive breast carcinomas than in in situ carcinomas or non-neoplastic breast epithelial cells. J Pathol, 195, 156-62.

Subramaniam D, Ponnurangam S, Ramamoorthy P, et al (2012). Curcumin induces cell death in esophageal cancer cells through modulating notch signaling. PLoS One, 7, e30590.

Sufi SA, Adigopula LN, Syed S, et al (2017). In-silico and in-vitro anti-cancer potential of a curcumin analogue (1E, 6E)-1, 7-Di (1H-Indol-3-Yl) Hepta-1, 6-Diene-3, 5-Dione. Biomed Pharm, 85, 389-98.

Sun GG, Wang Y, Chen LQ, et al (2011). Novel cancer suppressor gene for esophageal cancer: Manganese superoxide dismutase. Dis Esophagus, 24, 346-53.

Tang Y, Katuri V, Dillner A, et al (2003). Disruption of transforming growth factor-beta signaling in ELF beta -spectrin-deficient mice. Science, 299, 574-77.

Thapliyal R, Maru GB (2001). Inhibition of cytochrome P450 isozymes by curcumins in vitro and in vivo. Food Chem Toxicol, 39, 541-47.

Tian F, Min S, Xu P, Liu H, Xue L (2008). Curcumin promotes apoptosis of esophageal squamous carcinoma cell lines through inhibition of NF-KappaB signaling pathway. Chin J Cancer, 27, 566-70.

Tian F, Zhang C, Tian W, Jiang Y, Zhang X (2012). Comparison of the effect of P65 SiRNA and curcumin in promoting apoptosis in esophageal squamous cell carcinoma cells and in nude mice. Oncol Rep, 28, 232-40.

Tian F, Yang X, Liu Y, et al (2017). Constitutive activated STAT3 is an essential regulator and therapeutic target in esophageal squamous cell carcinoma. Oncotarget, 8, 88719-29.

Wang J, Zhang J, Zhang C, et al (2016). In situ proteomic 
profiling of curcumin targets in HCT116 colon cancer cell line. Sci Rep, 6, 22146.

Wang Y, Zhou P, Qin S, et al (2018). The curcumin analogs 2-Pyridyl Cyclohexanone induce apoptosis via inhibition of the JAK2-STAT3 pathway in human esophageal squamous cell carcinoma cells. Front Pharmacol, 9, 820.

Ye F, Zhang G, Guan B, Xu X (2012). Suppression of esophageal cancer cell growth using curcumin, (-)-epigallocatechin-3gallate and lovastatin. World J Gastroenterol, 18, 126-35.

Yu H, Lee H, Herrmann A, Buettner R, Jove R (2014). Revisiting STAT3 signalling in cancer: New and unexpected biological functions. Nat Rev Cancer, 14, 736-46.

Yu H, Jove R (2004). The STATs of cancer - new molecular targets come of age. Nat Rev Cancer, 4, 97-105.

Zhang F, Zhang H, Liu Y, Huang Y (2015). Curcumin inhibits Ec109 cell growth via an AMPK-mediated metabolic switch. Life Sci, 134, 49-55.

Zheng B, Liu T, Chen G, Zhang J, Kang X (2018). The effect of curcumin on cell adhesion of human esophageal cancer cell. Eur Rev Med Pharmacol Sci, 22, 551-60.

\section{(c) (i) (8)}

This work is licensed under a Creative Commons AttributionNon Commercial 4.0 International License. 